\title{
Modeling Protein Interaction Networks with Answer Set Programming
}

\author{
Timur Fayruzov*, Martine De Cock ${ }^{* \dagger}$, Chris Cornelis* and Dirk Vermeir ${ }^{\ddagger}$ \\ ${ }^{*}$ Dept. of Applied Mathematics and Computer Science, Ghent University, Krijgslaan 281 (S9), 9000 Ghent, Belgium \\ Email: firstname.lastname@ugent.be \\ ${ }^{\dagger}$ Institute of Technology, University of Washington, Tacoma, WA-98402, USA \\ Email: mdecock@u.washington.edu \\ ${ }^{\ddagger}$ Department of Computer Science, Vrije Universiteit Brussel, Pleinlaan 2, 1050 Brussels, Belgium \\ Email: dvermeir@tinf.vub.ac.be
}

\begin{abstract}
In this paper we propose the use of answer set programming (ASP) to model protein interaction networks. We argue that this declarative formalism rivals the popular boolean networks in terms of ease of use, while at the same time being more expressive. As we demonstrate for the particular case of a fission yeast network, all information present in a boolean network, as well as relevant background assumptions, can be expressed explicitly in an answer set program. Moreover, readily available answer set solvers can then be used to find the stable states of the network.
\end{abstract}

Keywords-Biological system modeling, Logic, Artificial intelligence

\section{INTRODUCTION}

With the increasing availability of experimental biological information, biological systems modeling has become an important research domain. Existing approaches (see e.g. [5], [8] for good overviews) can roughly be divided into two groups. Quantitative models are mostly made up of various kinds of differential equations, and considerable efforts are required to build such models. A lot of experimental data, such as the concentration of different types of molecules at different time points, is needed. This information is often very difficult and expensive to obtain.

Qualitative models are less information-demanding, but less precise as well. However, it turns out that significant simplifications, such as using a discrete timing and disregarding quantitative information, still allow to model the behavior of a system correctly. Thus, many efforts have been made to adopt discrete modeling techniques. Among them, discrete dynamical networks based on boolean networks are one of the best established qualitative modeling methods widely used by biologists to model protein regulation networks (see e.g. [1], [4]). The nodes of a boolean network represent protein molecules and the directed edges represent interactions. Edges can be typed to represent different kinds of interactions, such as inhibition and activation.

While liked for their simplicity, dynamical networks have the disadvantage of not being self-descriptive, i.e. they are built under some background assumptions that are not explicitly stated in the network itself. Furthermore, as explained in Section II-B, their use requires the development
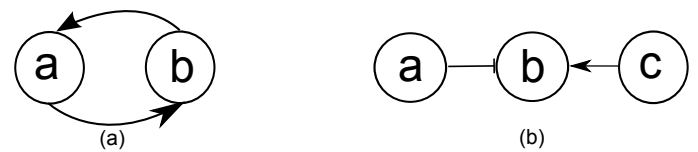

(b)

Figure 1. Examples of boolean networks

of specific algorithms to retrieve the stable states of the network (see e.g. [9]), and different algorithms can cause different execution flows.

In this paper, we propose to represent protein interaction networks by answer set programs [10]. As a first illustration, Example 1 shows an answer set program corresponding to the network depicted in Figure 1a. The prefixes $G$ and $S$ are used to separate general rules from specific rules describing a particular network. Rules S1-S4 capture all the information that is present in Figure 1a, namely that $a$ and $b$ are proteins (rules $\mathrm{S} 1$ and $\mathrm{S} 2$ ), that $a$ activates $b$ at any given time $T$ (rule S3), and likewise that $b$ activates $a$ (rule S4). Rules S5 and S6 express that in the initial state (time step 0), $a$ is active while $b$ is not. The G-rules describe a general boolean network semantics that does not change from network to network; we refer to Section III for more details.

Example 1 Answer set program $P_{1}$ for the network in Figure 1a.

$$
\begin{aligned}
& \text { G1 : } \quad \text { time }(0 . .2) \\
& \text { G2: } \quad \operatorname{act}(Y, T+1) \leftarrow \operatorname{act}(X, T), \operatorname{activates}(X, Y, T) . \\
& \text { G3: } \operatorname{inh}(Y, T+1) \leftarrow \operatorname{act}(X, T), \text { inhibits }(X, Y, T) \text {. } \\
& G 4: \quad \leftarrow \quad \operatorname{act}(X, T), \operatorname{inh}(X, T) \text {. } \\
& \text { G5: } \operatorname{act}(X, T+1) \leftarrow \operatorname{act}(X, T), \operatorname{not} i n h(X, T+1) . \\
& G 6: \quad \operatorname{inh}(X, T+1) \leftarrow \operatorname{inh}(X, T), \operatorname{not} \operatorname{act}(X, T+1) . \\
& S 1: \operatorname{prot}(a) . \quad S 4: \operatorname{activates}(b, a, T) \text {. } \\
& S 2: \operatorname{prot}(b) . \quad S 5: \operatorname{act}(a, 0) \text {. } \\
& \text { S3: activates }(a, b, T) . \quad S 6: \quad \operatorname{inh}(b, 0) \text {. }
\end{aligned}
$$

As explained below, the answer set of $P_{1}$ is $\langle\operatorname{act}(a, 0)$, $\operatorname{inh}(b, 0), \operatorname{act}(a, 1), \operatorname{act}(b, 1), \operatorname{act}(a, 2), \operatorname{act}(b, 2), \operatorname{act}(a, 3)$, $a c t(b, 3)\rangle$. Here predicates correspond to a protein state at a corresponding time step. In this answer set there is no difference between the protein states at time steps 1 and 2 . Hence we conclude that, under the given initial conditions, the stable state of the system is $\langle\operatorname{act}(a), \operatorname{act}(b)\rangle$. The actual answer set of the program includes more information that 
is not relevant to our task. In our answer set representation we omit this information for the sake of conciseness.

Note that only network specific rules such as rules S1S6 need to be redefined for a given protein interaction network, while the other rules model general biological properties. This makes the representation of such networks in ASP intuitively simple, while at the same time the ASP machinery becomes available to analyze and predict the behavior of the described network at hand. One of the main advantages of modeling with ASP is that all background information can and should be expressed explicitly in the model, while it is implicit in dynamical networks. This also allows to normalize different networks into one standard form. Furthermore, the use of ASP eliminates the need for specific network execution algorithms to retrieve the stable states of the networks. In fact, another main advantage of using ASP is that all supporting tools such as solvers and grounders are readily available. For the results described in this paper we used the lparse [14] grounder coupled with the smodels [12] solver, which is a default choice for many ASP-related tasks.

Our work is not the first attempt to use ASP to model biological networks. In [2], [6], [15] the authors propose to use ASP-based action languages to model, query or plan the execution of biological systems. Our approach, however, is different in several aspects that are discussed in Section V.

The paper is structured as follows. We begin by recalling the necessary preliminaries about ASP and boolean networks in Section II. In Section III we explain in detail how to describe a protein interaction network as an answer set program: we develop a framework of general rules (Grules), that describe a general boolean network semantics, and we give examples of specific rules (S-rules), that allow a biologist to describe a specific protein interaction network under study. In Section IV we illustrate our approach on a fission yeast network, while in Section $\mathrm{V}$ we explain its relationship with existing work. We conclude in Section VI.

\section{PRELIMINARIES}

\section{A. ASP}

Answer set programming [10] is a declarative formalism that allows to express relations between truth values of propositions with rules of the form

$$
L_{0} \leftarrow L_{1}, \ldots, L_{m}, \text { not } L_{m+1}, \ldots, \text { not } L_{n}
$$

in which $L_{0}, L_{1}, \ldots, L_{n}$ are called literals. The left-hand side of a rule is called the head, while the right-hand side is called the body of the rule. A rule with an empty head, such as rule G4 from Example 1, is called a constraint. A rule with an empty body is called a fact (rules S1-S6). A rule intuitively states that whenever literals in the body hold true, proposition head should be true as well. A literal can be negated, then it is preceded by the symbol $\neg$ and is called a negative literal. Another form of negation that represents a special feature of ASP, is negation-as-failure (naf) denoted by not. For instance, rule G5 from Example 1 states that if protein $X$ is active at time $T$ and there is no evidence that $X$ becomes inhibited at time $T+1$, then $X$ should still be active at time $T+1$.

An answer set program is a set of rules such as program $P_{1}$ from Example 1. The set of all literals of a program $P$ is denoted by Lit $_{P}$. An interpretation of $P$ is any consistent ${ }^{1}$ subset $S \subseteq \operatorname{Lit}_{P} . S$ is said to satisfy a rule with a nonempty head, if $\left\{L_{1}, \ldots, L_{m}\right\} \subseteq S$ and $\left\{L_{m+1}, \ldots, L_{n}\right\} \cap S=\emptyset$ implies that $L_{0} \in S$. When the head is empty, then $S$ is said to satisfy the rule if $\left\{L_{1}, \ldots, L_{m}\right\} \nsubseteq S$ or $\left\{L_{m+1}, \ldots, L_{n}\right\} \cap S \neq \emptyset$.

An interpretation that satisfies all rules of a program $P$ is called a model of $P$. Answer sets are special kinds of models. First of all, for a program $P$ without naf, an answer set of $P$ is a minimal model of $P$, i.e. $S$ is called an answer set of $P$ iff $S$ is a model of $P$ and there is no model $K$ such that $K \subset S$.

Example 2 The models of

$$
a \leftarrow b
$$

are $\langle a, b\rangle,\langle a\rangle$ and $\emptyset$. The minimal model is $\emptyset$.

The concept of an answer set is extended for the program $P$ containing negation-as-failure as described below. Suppose that $S$ is a model of $P$, and our hypothesis is that $S$ is an answer set of $P$. In order to check if $S$ is an answer set of $P$, we build a reduct program $P^{\prime}$ by 1 ) removing from $P$ all rules that contain a naf-literal not $L$, with $L \in S$;2) removing all the naf-literals from the bodies of the remaining rules. If the minimal model $S^{\prime}$ of the naf-free program $P^{\prime}$ coincides with $S$, then $S$ is an answer set of the original program $P$.

Example 3 A program with negation-as-failure can have more than one answer set. Suppose that we have one seat and two persons $a$ and $b$, and we want to assign the seat to one of them. We can model this by the following program

$$
\begin{aligned}
& \operatorname{seat}(a) \quad \text { not seat }(b) \\
& \operatorname{seat}(b) \leftarrow \operatorname{not} \operatorname{seat}(a)
\end{aligned}
$$

It has two answer sets $\langle\operatorname{seat}(a)\rangle$ and $\langle\operatorname{seat}(b)\rangle$.

\section{B. Dynamical networks}

A dynamical network of protein interactions captures interactions between proteins in the form of a graph. Nodes represent proteins and edges represent interactions between proteins. The state of a network is determined by the state of every protein in the network, e.g. the on/off state in the boolean case. Every node has input nodes that are

\footnotetext{
${ }^{1}$ The set is said to be consistent if it does not contain literals $a$ and $\neg a$ together
} 
determined by inbound edges, and output nodes that are determined by the outbound edges of the node. For example in Figure 1a $b$ is at the same time an input and output node for node $a$. For every node in the network a transition function is defined that determines the next state of the node depending on the node's inputs. The network can switch between states by applying these functions on its nodes. The update can occur synchronously (all elements are updated simultaneously) or asynchronously (one or several nodes are updated at once). A sequence of network states obtained by such transitions is called a trajectory of the system.

Example 4 Assume, that in Figure 1a protein $a$ is active and $b$ is inhibited, then the initial network state will be $\langle 1,0\rangle$. We have 2 transition functions: $a \rightarrow b$ and $b \rightarrow a$ (reads as 'if a is 1 , then $b$ is 1 ' and 'if $b$ is 1 , then a is 1 ' correspondingly). By applying these functions to the initial state, we can go to the next network state $\langle 1,1\rangle$. If we apply the transition functions once again, the state does not change any more which means that the network has reached a stable state. A trajectory of the network in this case is $\langle 1,0\rangle,\langle 1,1\rangle$.

The number of states of a network is finite, so the number of different states in a trajectory is finite as well. Because a boolean network is deterministic, every trajectory will reach a stable state (point attractor) or stable cycle (dynamic attractor). A set of trajectories that reach the same attractor is called a basin of attraction. For example a basin of attraction for the state $\langle 1,1\rangle$ of the network in Figure 1a consists of two trajectories: $\langle 0,1\rangle,\langle 1,1\rangle$ and $\langle 1,1\rangle$ itself.

\section{BUILDING A NETWORK MODEL IN ASP}

In this section, we set up the framework for describing protein interaction networks as answer set programs. We begin with a detailed explanation of the G-rules of $P_{1}$ in Example 1. Next we deal with issues such as conflicts and self-degradation that do not occur in the network of Figure 1a but might occur in other protein interaction networks.

\section{A. Describing entities and their influences}

The first step in describing a protein network is to introduce the proteins, their initial states as well as their interactions, cfr. rules S1-S6 in Example 1. By themselves these rules do not model anything; although they define the connection between proteins, they do not describe the influence of these connections on the proteins at the different time steps - this is the task of G-rules. First of all, rule G1 is merely a shorthand for the facts

$$
\text { time(0). time(1). time(2). }
$$

to introduce time steps into the program. Rules G2 and G3 define the actual semantics of the activation and inhibition concepts. $X$ and $Y$ in these rules are variables, that are substituted by actual proteins (such as $a, b$ ) during program execution. The activation rule G2 for example, says that protein $Y$ will be active at time step $T+1$ if protein $X$ is active and there is an activating connection between $X$ and $Y$ at time step $T$.

Rule G4 is a constraint that expresses that a protein can not be active and inhibited at the same time. Rules G5 and G6 are inertia rules that express what happens to a protein when there is no external influence: at the next time step a protein retain its state unless it was changed.

The task of an ASP solver is to find answer sets. In our application scenario, an answer set contains a sequence of protein states for each time point (see e.g. Example 1). Therefore, we can retrieve the stable state of the network by looking at the protein configurations in an answer set at each time step. When the configuration in two consequent time steps does not change, a stable state has been reached. In Example 1 we reach the stable state at time point 1, because the protein states do not change after this point. Note that, even though the last time step in rule G1 in Example 1 is 2, the network evolution is computed until time step 3 because the heads of the rules of program $P_{1}$ contain $T+1$.

\section{B. Resolving conflicts}

Rules G2-G4 might fail to work for more complex protein interaction networks. Below we explain why they should be replaced with more refined rules, as well as supplemented by supporting rules. The approach described so far does not allow to generate any answer set for the network in Figure $1 \mathrm{~b}$ because protein $b$ is being inhibited and activated at the same time, which violates the integrity constraint expressed as rule G4. To resolve this conflict, we adopt the solution used in [4]: if there are more incoming activation links than inhibition links, then the protein is active; if there are more inhibition links, then the protein is inhibited; if their number is equal, then the protein retains the previous state. Furthermore, we introduce the notion of inhibition and activation thresholds to allow more flexibility for describing protein behavior. Let us return to Figure $1 b$. Under the current definitions, protein $b$ does not change its state when both $a$ and $c$ are active, i.e. if $b$ is active it remains active. Suppose now that we want to modify the behavior of $b$ to change its sensitivity to the activating or inhibiting influence such that it requires less effort (less activation/inhibition inputs) to change the state of the protein. This requirement can be implemented in the system by introducing inhibition/activation levels. To implement this, we need to adjust the constraint as well as the activation and inhibition rules. The superscript in the rule labels below denotes the version of the rules; the complex numeration denotes the supporting rules for the main rule.

$$
\begin{aligned}
& G 2^{1}: \operatorname{act}(Y, T+1) \leftarrow \operatorname{act}(X, T), \operatorname{activates}(X, Y, T), \\
& \text { not conflict }(Y, T) \text {, } \\
& \text { not mod_act_th }(Y) \text {. } \\
& \text { G2.1: } \operatorname{act}(Y, T+1) \leftarrow \operatorname{conflict}(Y, T), \operatorname{act} \_t h(Y, T h), \\
& \text { \#_act }(Y, A, T) \text {, \#_inh }(Y, I, T) \text {, } \\
& A-I>T h \text {. }
\end{aligned}
$$




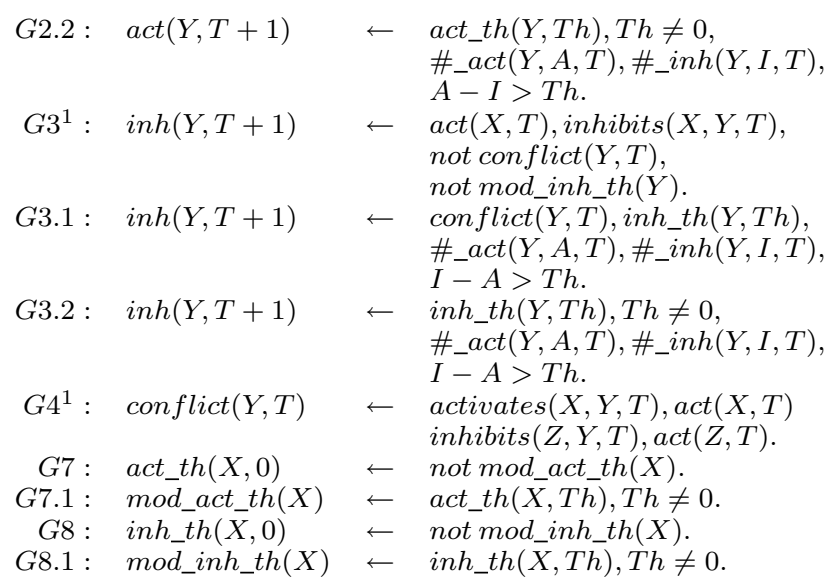

Rules G1,G5 and G6 are omitted because they do not change. The other rules state that if there is no conflict and thresholds are default, then the old definitions work (rules $\mathrm{G} 2^{1}$ and $\mathrm{G} 3^{1}$ ), but if there is a conflict (the body of rule $\mathrm{G} 4^{1}$ is satisfied), then we count the number of activation and inhibition links for the conflicting instance and make the decision based on this count and on the activation and inhibition thresholds of the protein (rules G2.1 and G3.1). Moreover, if there is no conflict but a corresponding threshold was changed, we follow rules G2.2 and G3.2 in the same fashion. The integrity constraint G4 we had before is now transformed to the definition of conflict (rule $\mathrm{G} 4^{1}$ ). It fires only if there are inhibits and activates links on the protein and both can be executed at the current time point. The definition of the \#_act and \#_inh predicates is omitted here because of space limitations, but can be found in [7]. Rules G7 and G8 set the activation and inhibition threshold of every protein to 0 in case it was not set explicitly (G7.1 and G8.1). Having both inhibiting and activating thresholds instead of one threshold is not redundant, since these thresholds characterize not the 'on/off' level of the protein, but rather an effort that is needed to change its state. The thresholds can be viewed as tolerance degrees of a protein to a corresponding input. Positive values make the protein more tolerant and negative make it less tolerant. The default value can be changed as illustrated below.

Example 5 Let $P_{3}$ be the answer set program consisting of general rules $\mathrm{G} 1, \mathrm{G} 2^{1}$, G2.1, G2.2, G3 ${ }^{1}$, G3.1, G3.2, G4 ${ }^{1}$, G5, G6, G7, G7.1, G8, G8.1 and the specific rules:

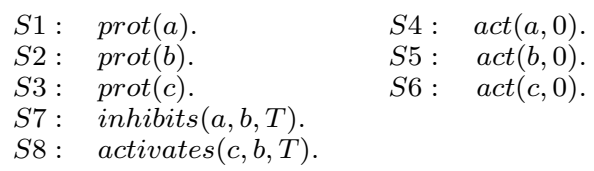

corresponding to the network in Figure 1b. The activation and inhibition thresholds of these proteins are not explicitly defined; hence they are automatically set to the default value. The answer set of this program is $\langle\operatorname{act}(a, 0), \operatorname{act}(b, 0)$, $\operatorname{act}(c, 0) \operatorname{act}(a, 1), \quad \operatorname{act}(b, 1), \quad \operatorname{act}(c, 1), \quad \operatorname{act}(a, 2), \operatorname{act}(b, 2)$, $\operatorname{act}(c, 2), \operatorname{act}(a, 3), \operatorname{act}(b, 3), \operatorname{act}(c, 3)\rangle$. The state of protein $b$ does not change over time since its inhibiting and activating inputs are equal, and its thresholds for activation and inhibition are both 0 . From the answer set we retrieve that the stable state is $\langle\operatorname{act}(a), \operatorname{act}(b), \operatorname{act}(c)\rangle$.

Example 6 For the network in Figure 1b, let us explicitly set the inhibition threshold of $b$ to -1 to indicate that this protein is susceptible to inhibition. In other words, let $P_{4}$ be the answer set program containing all the rules from $P_{3}$ as well as the additional rule S9: inh_th $(b,-1)$. The answer set of this program is $\langle\operatorname{act}(a, 0), \operatorname{act}(b, 0), \operatorname{act}(c, 0) \operatorname{act}(a, 1)$, $\operatorname{inh}(b, 1), \quad \operatorname{act}(c, 1), \quad \operatorname{act}(a, 2), \quad \operatorname{inh}(b, 2), \quad \operatorname{act}(c, 2), \operatorname{act}(a, 3)$, $\operatorname{inh}(b, 3), \operatorname{act}(c, 3)\rangle$. The stable state in this case is $\langle\operatorname{act}(a)$, $\operatorname{inh}(b), \operatorname{act}(c)\rangle$.

The phenomena of self-activation and self-degradation can also be solved by adjusting activation and inhibition thresholds. Self-activation/degradation means that a protein is able to change its state when no external influence is applied. Let us consider the following example:

Example 7 Let $P_{5}$ be the AS program containing all the rules from $P_{3}$, but rules $\mathrm{S} 4-6$ are replaced with the following:

$$
\text { S4: } \quad \operatorname{inh}(a, 0) . \quad S 5: \quad \operatorname{inh}(b, 0) . \quad S 6: \quad \operatorname{inh}(c, 0) .
$$

to indicate that all proteins in Figure $1 \mathrm{~b}$ are initially inhibited. Furthermore, we add the additional rule rule S9: act_th $(b,-1)$. to indicate that $b$ is susceptible to activation. According to the rule $\mathrm{G} 2.2$, in this case protein $b$ activates itself when no inhibition influence is applied.The answer set of program $P_{5}$ is $\langle\operatorname{inh}(a, 0), \operatorname{inh}(b, 0), \operatorname{inh}(c, 0) \operatorname{inh}(a, 1)$, $\operatorname{act}(b, 1), \operatorname{inh}(c, 1), \operatorname{inh}(a, 2), \operatorname{act}(b, 2), \operatorname{inh}(c, 2)\rangle$. The stable state in this case is $\langle\operatorname{inh}(a), \operatorname{act}(b), \operatorname{inh}(c)\rangle$.

\section{Starting conditions}

By writing S-rules, a user can model various networks and observe their behavior under certain initial conditions. This requires the user to consequently set various initial protein activation combinations and analyze the results of each execution. On large networks with tens of proteins, the number of different possible combinations is very high, which makes this task very cumbersome. To automate this process, we introduce two additional general rules that deal with different initial condition combinations:

$$
\begin{aligned}
& G 9: \operatorname{act}(X, 0) \leftarrow \operatorname{not} i n h(X, 0) . \\
& \text { G10: } \operatorname{inh}(X, 0) \leftarrow \operatorname{not} \operatorname{act}(X, 0) \text {. }
\end{aligned}
$$

These rules force the solver to make a choice for each protein: either it is active at the initial time point or inhibited. In this way, different answer sets are automatically generated for each possible starting combination, which decreases the need for manual input of the user drastically.

\section{ANSWER SET PROGRAM FOR THE FISSION YEAST NETWORK}

In this section we describe a proof-of-concept experiment for the analysis of a real interaction network. We take 


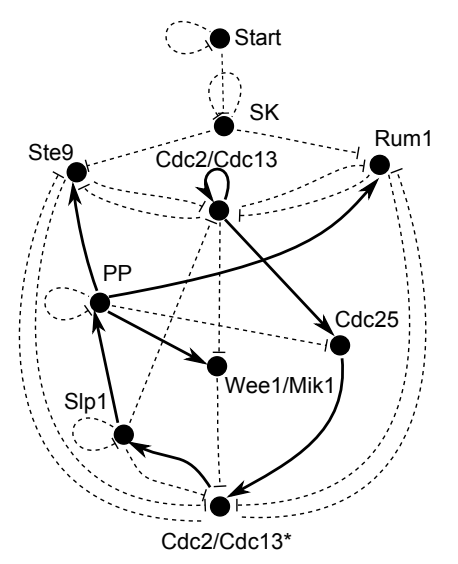

Figure 2. The dynamical network model of the Fission Yeast cell cycle. Taken from [4].

the dynamical network model of the Fission Yeast network described in [4], and translate it to our framework. The structure of the network is depicted in Figure 2. The authors of [4] report that the network contains 12 stable states as well as 1 stable cycle consisting of 3 states.

To analyze the network, we include all G-rules described above in the framework, namely: G1, G2 ${ }^{1}, \mathrm{G} 2.1, \mathrm{G} 2.2, \mathrm{G} 3^{1}$, G3.1, G3.2, G41 , G5, G6, G7, G7.1, G8, G8.1, G9, G10. Note that this set of rules defines the network semantics that is independent of any particular network such as Fission Yeast. This means, that if we want to build another network where proteins show the same behavior patterns (such as e.g. self-inhibition/activation) we can use the framework without any modifications.

The network specific part of the program is captured in Srules. This part consists of rules like activates (PP, Ste9), act_th $(C d c 2 / C d c 13,-1)$, etc. The full listing of the program can be found in [7]. Some facts such as the activation threshold of $C d c 2 / C d c 13$ or the inhibition threshold of $C d c 2 / C d c 13^{*}$ are not explicitly seen on the network in Figure 2; to figure out the exact execution flow one has to read the corresponding article [4]. This shows the implicit advantage of an ASP model in comparison with boolean networks. The ASP model is self-descriptive, i.e. it does not rely on any implicit assumptions or background knowledge, while with boolean networks, the user needs to check the conditions for every node before execution.

In Table I we provide the results for the network trajectory from an initial state that corresponds to the point of cell division initiation. The table is a structured representation of the derived answer set. Rows stand for proteins, and columns stand for the time flow, i.e. one row shows the changes of the protein over time. We denote an act state with 1 in the appropriate cell and inh with 0 . For example, $\operatorname{act}(S K, 0)$ converts to 1 in the row SK and column 0. Execution shows that after the 8th step the system has reached a stable state.
Table I

NETWORK EXECUTION FLOW

\begin{tabular}{l|c|c|c|c|c|c|c|c|c|c|c} 
Protein & 0 & 1 & 2 & 3 & 4 & 5 & 6 & 7 & 8 & 9 & 10 \\
\hline Start & 1 & 0 & 0 & 0 & 0 & 0 & 0 & 0 & 0 & 0 & 0 \\
SK & 0 & 1 & 0 & 0 & 0 & 0 & 0 & 0 & 0 & 0 & 0 \\
Cdc2/Cdc13 & 0 & 0 & 0 & 1 & 1 & 1 & 1 & 0 & 0 & 0 & 0 \\
Ste9 & 1 & 1 & 0 & 0 & 0 & 0 & 0 & 0 & 1 & 1 & 1 \\
Rum1 & 1 & 1 & 0 & 0 & 0 & 0 & 0 & 0 & 1 & 1 & 1 \\
Slp1 & 0 & 0 & 0 & 0 & 0 & 0 & 1 & 1 & 0 & 0 & 0 \\
Cdc2/Cdc13* & 0 & 0 & 0 & 0 & 0 & 1 & 1 & 0 & 0 & 0 & 0 \\
Wee1/Mik1 & 1 & 1 & 1 & 1 & 0 & 0 & 0 & 0 & 1 & 1 & 1 \\
Cdc25 & 0 & 0 & 0 & 0 & 1 & 1 & 1 & 1 & 0 & 0 & 0 \\
PP & 0 & 0 & 0 & 0 & 0 & 0 & 0 & 1 & 1 & 0 & 0
\end{tabular}

This trajectory and the resulting stable state has also been found in [4]. In fact, there is a one-to-one correspondence between our results and those reported in [4].

\section{RELATED WORK}

To the best of our knowledge, all approaches that adopt ASP to model biological phenomena are based on the concept of action languages. An action language is a highlevel language with a simple intuitive syntax that allows to describe the states of the world and effects of actions on these states [11]. The statements and queries in this language are then translated to standard ASP syntax and executed to find the answer sets.

The first attempt to model biological processes by means of ASP was made by Baral et al. in [2] where they present a BioSigNet-RR system that allows to represent and reason about signal networks. In this paper they define an action language that allows to express the structure of a signal network (they use $N F \kappa B$ network as an example) as well as the means to query the network (does protein $a$ bind to $b$ ?), plan the execution (what sequence of actions should be taken to make $a$ active?) and explain the results (given that $a$ is active at a certain point, what was the initial state?). This work was further extended by Dworschak et al. in [6] who introduced several extra concepts, such as allowance and noconcurrency, as well as a special language for biological modeling called $C_{T A I D}$.

It is important to emphasize the difference between action language-based approaches [2], [6] and the approach we propose here. The former requires that for each model the whole program is built from scratch and every interaction is defined as its own rule. In other words, the framework describes only the description language, and the biologist has to describe every interaction separately. In our setting we go one step further, and provide the biologist with a background theory based on a boolean network model semantics. For example, let us consider an example where protein $b$ is activated by $a$ and $c$ and inhibited by $d$. In the default boolean network semantics, if the number of activating links is higher that the number of inhibition links, then protein $b$ will be activated, if it is lower it will be inhibited, if it is equal, the state remains unchanged. To express this 
with action languages we need 2 actions: activating_prot_b, inhibiting_prot_b, and the following set of statements:

$\begin{array}{lll}\text { activating_prot_b } & \text { causes } & b \\ \text { inhibiting_prot_b } & \text { causes } & \neg b \\ a, \neg d & \text { causes } & \text { activation_prot_b } \\ c, \neg d & \text { causes } & \text { activation_prot_b } \\ a, c & \text { causes } & \text { activation_prot_b } \\ d, \neg a, \neg c & \text { causes } & \text { inhibiting_prot_b }\end{array}$

The number of required rules grows combinatorially with the number of interactions.

In the framework that we propose, only factual input is needed from the biologist: activates $(a, b)$, activates $(c, b)$, inhibits $(d, b)$. The boolean network semantics implemented in the framework will do all other necessary inference. Moreover, as ASP offers non-monotonic inference, the existing semantics can be extended with additional features when needed, making the framework scalable.

A detailed analysis of other related approaches to model biological networks is provided in [15]. A large group of such approaches uses process calculi theory (see e.g. [3]) in which molecules are represented as processes, and interactions are represented as communication channels between processes. Another group uses Petri Nets (see e.g. [13]), a computer science formalism that features concurrency and has been adopted to build biological networks.

The approaches described in the previous paragraph follow a simulation-perturbation strategy, i.e. to analyze the behavior of a system, the biologist changes the input and observes the changes on the output. Even though the structure of the system is known, it still works as a blackbox in the sense that the result cannot be explained. As a result, many computationally expensive experiments may be needed to explain some properties of the model. ASP-based methods aim to overcome this limitation.

\section{CONCLUSIONS AND FUTURE WORK}

In this paper, we have proposed to model protein interaction networks as answer set programs. Answer set programming (ASP) is an area of logic programming that allows to model systems that exhibit nonmonotone behavior using negation-as-failure. These models, represented as programs, can be executed to produce the set of stable states of a given protein interaction network.

Our ASP model has the advantage of being more formal compared to boolean networks, since it requires that all implicit assumptions and background knowledge be explicitly described in the body of the program, while in boolean networks this knowledge can be hidden in the non-formal description. The approach remains however straightforward to apply; it does not require any formal logics knowledge from the biologist, who can operate with ready-to-apply blocks to build a model. At the same time the approach is very flexible due to the fact that nonmonotonic reasoning is used and any specific case which does not fit in a general picture can be incorporated with a minimal effort. Moreover, readily available answer set solvers can be used to find the stable states of a network.

Although the framework extends the boolean network functionality, it inherits some of its limitations as well. One of such limitations (which is inherent in most discrete modeling formalisms) is that every action should happen within one time step. In other words, it is impossible to model slow and fast interaction; everything is aligned to the same speed. Moreover, in the current version of the system only synchronous execution is supported. However, we intend to extend the framework with the possibility to handle asynchronous cases as well.

\section{REFERENCES}

[1] R. Albert. Boolean modeling of genetic regulatory networks. In Complex Networks, pages 459-481. 2004.

[2] C. Baral, K. Chancellor, N. Tran, N. Tran, A. M. Joy, and M. E. Berens. A knowledge based approach for representing and reasoning about signaling networks. Bioinformatics, 20 Suppl 1, 2004.

[3] F. Ciocchetta and J. Hillston. Process algebras in systems biology. In SFM, 2008.

[4] M. I. Davidich and S. Bornholdt. Boolean network model predicts cell cycle sequence of fission yeast. PLOS ONE, 3(2), 2008

[5] H. de Jong. Modeling and simulation of genetic regulatory systems: A literature review. Journal of Computational Biology, 9(1):67-103, 2002.

[6] S. Dworschak, S. Grell, V. J. Nikiforova, T. Schaub, and J. Selbig. Modeling biological networks by action languages via asp. Constraints, 13(1-2):21-65, 2008.

[7] T. Fayruzov. Technical report 2009-01. Technical report, Ghent University, 2009. http://www.cwi.ugent.be.

[8] J. Fisher and T. A. Henzinger. Executable cell biology. Nature Biotechnology, 25(11):1239-1249, November 2007.

[9] A. Garg, A. D. Cara, I. Xenarios, L. Mendoza, and G. D. Micheli. Synchronous vs asynchronous modeling of gene regulatory networks. Bioinformatics, 24(17):1917-1925, 2008.

[10] M. Gelfond and V. Lifschitz. The stable model semantics for logic programming. In ICLP/SLP. MIT Press, 1988.

[11] M. Gelfond and V. Lifschitz. Representing actions in extended logic programming. In JICSLP, 1992.

[12] I. Niemelä and P. Simons. Smodels - an implementation of the stable model and well-founded semantics for normal lp. In $L P N M R, 1997$.

[13] M. Peleg, I. Yeh, and R. B. Altman. Modelling biological processes using workflow and petri net models. Bioinformatics, 18(6):825-837, 2002.

[14] T. Syrjänen. Lparse 1.0. User's manual.

[15] N. Tran. Reasoning and hypothesing about signaling networks. PhD thesis, Arizona State University, December 2006. 